\title{
Effect of neodymium nanoparticles on optical properties of zinc tellurite glass system
}

\begin{abstract}
A system of zinc tellurite glasses with chemical composition [(TeO2)0.70 ( $\mathrm{ZnO}) 0.30] 1-\mathrm{x}(\mathrm{Nd} 2 \mathrm{O} 3$ NPs)x, where $\mathrm{x}$ ranged from 0.01 to 0.05 molar fraction have been prepared and coded as TZND have been fabricated using the melt-quenching technique. The aim was to study the structural, physical, and optical properties of the sample glasses. The choice of the chemical composition was to provide high refractive index, favourable metallization criterion and other optical qualities for linear and non-linear optical applications. The optical properties were studied using the UV-Vis spectroscopic analysis. The density and molar volume were found to increase with Nd2O3 NPs concentration. The FTIR spectral analysis showed the existence of $\mathrm{TeO} 3$ and $\mathrm{TeO} 4$ structural units which varying concentrations of $\mathrm{TeO} 4$ observed with $\mathrm{Nd} 2 \mathrm{O} 3$ nanoparticles (NPs). The transmission electron microscopy (TEM) analysis showed the agglomeration of the Nd2O3 NPs in the morphological structure of the glasses. The variations of the optical parameters were mostly related to the variation in the structural nature of the glasses $(\mathrm{TeO} 3$ and $\mathrm{TeO} 4$ concentration changes). High refractive index value, ranging between 2.5470 and 2.6093, favourable metallization criterion value range of 0.3406 and 0.3535 as well as high polarizabilities and the optical basicity reveal that the glasses have high potential in optical fibre technology as well as optical non-linear (laser) applications.
\end{abstract}

